\title{
The relationship between the exercise capacity and somatotype components, body composition, and quadriceps strength in individuals with coronary artery disease
}

\author{
Melike Gültekin', Gülay Yeginoglu², Hasan Bingol ${ }^{3}$ \\ ${ }^{1}$ Department of Anatomy, Institute of Health Science, Karadeniz Technical University, Trabzon, Turkey \\ ${ }^{2}$ Department of Anatomy, Faculty of Medicine, Karadeniz Technical University, Trabzon, Turkey \\ ${ }^{3}$ Department of Therapy and Rehabilitation, Vocational School of Health Services, Mus Alparslan University, Muş, Turkey
}

Received: 2021-08-04.

Accepted: 2021-11-09

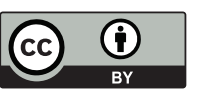

This work is licensed under a Creative Commons Attribution 4.0 International License

J Clin Med Kaz 2021; 18(6):62-68

Corresponding author:

Hasan Bingol.

E-mail:hesenbingol@gmail.com

/h.bingol@alparslan.edu.tr;

ORCID: 0000-0003-3185-866X

\section{Abstract}

Aim: The purpose of this cross-sectional study was to explore a possible relationship between exercise capacity and somatotype components, body composition, and quadriceps strength in individuals with coroner artery disease (CAD)

Material and methods: A convenient sample size of 60 participants between the ages of 45 and 60 years (mean age $56.6 \pm 4.7 ; 12$ females [mean age 57.7 \pm 3.01 ], 48 males [mean age; $56.3 \pm 5.1]$ was established from patients referred to the Department of the Cardiopulmonary Rehabilitation. The exercise was assessed using graded symptom-limited exercise testing while Participants' body composition was detected via Bioelectrical impedance analysis (BIA) technique by using TANITA SC-330 (TANITA, Tokyo, Japan). Finally, quadriceps femoris muscles testing was conducted with Dr. Robert W. Lovett's manual muscle testing method.

Results: In the whole sample, exercise capacity was found to be negatively and weakly correlated with the endomorph component $(r=-0.39)$, whereas a slight and positive correlation was observed between the ectomorph component and exercise capacity $(r=0.28)$. However, the correlations revealed between exercise capacity and somatotype components differentiated with gender-based analysis. A moderate and weak correlation was found out between exercise capacity and fat mass (FM) in the whole group and the male individuals ( $r=-0.45$ and $r 2=-0.34)$, respectively; in contrast, there was not a meaningful correlation between the same variables in the female individuals $(r=-0.002)$. Exercise capacity had slight and negative relationships with free-fat mass variable (FFM) in both gender ( $r=-0.28$ and $r 2=-0.29$ ), while small to moderate relationship with fat-mass variable (FM) in the male gender ( $r 7=-0.34)$. Body composition elements, including FM, FFM and BMI, together explained a \% 24 of variance in exercise capacity, while somatotype components together explained a \% 16 of variance in the exercise capacity. Finally, quadriceps femoris strength was found to be the best predictor of exercise capacity of a patient with CAD (R2=0.44 or \% 44)

Conclusion: Our results suggest that exercise capacity in individuals with CAD is directly associated with somatotype components, anthropometry/ body composition elements, and quadriceps strength.

Key words: aerobic capacity, fat mass index, body mass index, coronary artery disease, peak metabolic equivalent

\section{Introduction}

Coronary artery disease (CAD) is a significant cardiovascular disorder, which is the leading cause of death in both developed and developing countries [1]. Furthermore, because of both its most common causal factor of death in both genders and leading to diseases and socialeconomic burden, CAD is a significant health problem [2]. Although recently published reports have demonstrated promising decreases in the mortality rate associated with the $\mathrm{CAD}$, it remains a dominant causal factor for morbidity and mortality in many communities [3]. There are many risk factors for CAD, including both modifiable risk factors such as high blood pressure, high blood cholesterol level, smoking, diabetes, overweight or obesity, lack of physical activity, unhealthy diet, stress, and conventional risk factors such as age, sex, family history, and race [4]. In other words, 
biological, physiological, biochemical, environmental, and genetic risk factors are possible risk factors leading to a range of clinical phenotypes of cardiovascular diseases by interacting with one another [5]. Also, lifestyle has been found to play an essential role in developing such cardiovascular diseases [6]. The fact that the adiposity and muscularity are significantly associated with increased CAD [7] requires investigation of the possible relationship between somatotype components and coronary artery disease further. Previously, a possible relationship between somatotype components and anaerobic performance was reported in a cross-sectional study conducted by Ryan-Stewart et al. [8], indicating a significant correlation between somatotype ratings and anaerobic performance.

The relationship between physique and cardiovascular disease (CVD) has been previously investigated extensively; particularly, a possible relationship between body mass index (BMI) and coronary heart disease (CHD) has been frequently examined in cross-sectional studies $[3,9,10]$. A positive association was identified between morphological typology (somatotype) and muscle strength in children aged 10 to 11.5 years [11]. Ultimately, a relationship between body composition, peripheral muscle strength, and functional exercise capacity has been reported in a study by Hillman et al. [12], revealing that muscle strength and body mass might be predictive factors of functional exercise capacity. However, the body of evidence in this subject area is still insufficient and needs to be furthermore investigated. Additionally, aerobic capacity, described as a unit of metabolic equivalents (METs) or an estimate of the maximal oxygen uptake at a given workload, is one of the most common factors to predict a possible cardiovascular disease in healthy people $[13,14]$. As well, exercise capacity is known to be an important outcome for mortality in patients with cardiovascular disease as well as in healthy individuals [15]. Moreover, muscle strength, a significant component of physical fitness, has an essential role in preventing CAD. The existing evidence has suggested that muscle strength loss might lead to lower physical conditions and therefore reduced exercise capacity $[16,17]$.

Despite these abundant findings, a possible relationship between exercise capacity and somatotype, body composition, and quadriceps femoris muscle strength remains to be determined. Therefore, our focus in this observational study was to explore a possible association between exercise capacity and somatotype, body composition, and quadriceps femoris in individuals with CAD.

\section{Material and methods Subjects}

This current study was conducted in Cardiopulmonary Rehabilitation Unit of Trabzon Ahi Evren Thoracic and Cardiovascular Surgery Training and Research Hospital. The study protocol was approved by the Ethical Committee of the Faculty of Medicine of the Karadeniz Technical University for scientific research with the registration number of 24237859/187. The convenient sample size for this current cross-sectional study consisted of 60 individuals with CAD between the ages of 45 and 60 years (mean age; 56.6 $\pm 4.7: 12$ females [mean age; $57.7 \pm 3.01$ ], 48 males [mean age; $56.3 \pm 5.1$ ], who volunteered for this study. During the enrollment to the study, each patient was thoroughly informed about the study procedure, including testing procedures they would be gone through via illustrative and informative explanations. Furthermore, patients were asked to inform researchers whether they had a significant medical condition except for CAD. Also, each participant's medical doctor was asked whether their patients had a critical medical Journal of Clinical Medicine of Kazakhstan: 2021 Volume 18, Issue 6 condition preventing them from conducting testing procedures. After insightful explanations, written informed consent was obtained from all patients who accepted to participate in our study. This current study's process complied with the provisions of the Helsinki Declaration. Inclusion criteria: diagnosed with coronary artery disease by coronary angiography, at least three months after possible revascularization surgery, no habit of regular exercise, no smoking, using the same group of drugs, and having communication skills. Exclusion criteria: uncontrolled sinus tachycardia ( $>120$ beats/min), congestive heart failure, acute systemic disease or fever, orthostatic hypertension, uncontrolled diabetes (blood glucose $>400 \mathrm{mg} / \mathrm{dl}$ ), unstable angina, thrombophlebitis, new embolism, endocarditis, acute pericarditis, severe cardiac rhythm disturbances, severe LV dysfunction, aortic dissection, severe aortic stenosis, resting systolic blood pressure $>200 \mathrm{mmHg}$, resting diastolic blood pressure $>110 \mathrm{mmHg}$, uncontrolled atrial or ventricular arrhythmia, acute myocardial infarction (first two days) Left ventricular $\mathrm{EF}<50 \%$, severe orthopedic impairment preventing walking and running, and balance-visual-cognitive impairment.

\section{Measurements}

A physical therapist with ten years' experience conducted all measurements following standard procedures for each measurement under a senior medical doctor's supervision. The aim of the study was meticulously described to the patients who met inclusion criteria. Furthermore, before the assessment process, it was ensured that each participant was familiar with the testing procedures and equipment used. Finally, the reliability of the assessments was established by conducting several rehearsal assessments before the actual evaluation. There were no dropouts or adverse events throughout the study period.

\section{Exercise capacity}

The clinical exercise testing has been used to assess peak aerobic capacity to detect a potential risk of CAD for many years [18]. The evaluation of peak aerobic capacity was carried out using graded symptom-limited exercise testing, which incorporates a treadmill (GG treadmill), blood pressure module, pulse analyzer system, and an electrocardiographic monitor, according to the American heart association exercise testing guidelines [19]. All study participants were evaluated according to the Bruce ramp protocol, a valid measurement approach for maximal aerobic capacity where patients initiate exercising at $1.7 \mathrm{mph}$ per hour on a $10 \%$ grade [20]. In line with the Bruce protocol, the incline and speed of the treadmill were augmented every three minutes through a total of 7 phases. General principles for conducting the exercise testing regarding contraindications and reasons for stopping tests were established according to ACC/AHA guidelines for exercise testing by Gibbons et al. [19]. In accordance with this protocol, test sessions were terminated if the subjects displayed subjective unbearable symptoms (angina, dyspnea, and fatigue), abnormalities of rhythm and blood pressure, marked and progressive ST-segment deviation, or when they were not able to maintain [21]. Metabolic equivalent (MET), blood pressure (BP), and heart rate values (HR) were obtained throughout the testing sessions. The 12 lead ECG was being monitored continuously during the exercise test.

\section{Body composition assessment}

Anthropometric measurements are a widely accepted reliable method to provide knowledge about the body's size and proportion of body fat and muscles by measuring body width, length, skinfold thickness (SF), and circumference (C) 
[22]. Anthropometry was carried out on the right side of each participant's body in conformity with the previously reported guidelines [7]. Body composition was measured via Bioelectrical impedance analysis (BIA) technique, using TANITA SC-330 (TANITA, Tokyo, Japan), a useful tool for body composition analysis in healthy adults and children [23]. The following BIA variables were obtained: body weight, percent body fat $(\% \mathrm{BF} /$ FM), fat-free mass (FFM, kg). The body mass index (BMI) was calculated by dividing weight by the square of the subjects' height. Each participant was classified as "underweight," "normal weight," "overweight," and "obese," using the universal standard BMI equation [24].

\section{Manual muscle testing for knee extension}

Manual muscle testing is the most common test used as a reliable non-invasive and inexpensive method for assessing muscle strength in clinical settings [24]. In this study, quadriceps femoris (QF) muscles testing was conducted with Dr. Robert W. Lovett's manual muscle testing method. This test classifies muscle strength in six levels, with a higher level indicating normal power (5, normal, and 0 , total paralysis) [25]. Before the actual assessment, each participants was $\mathrm{c}$ informed about the test procedure in detail. The participant was seated on a chair with the hips and knees flexed at $90^{\circ}$, arms crossed on the chest, and was asked to straighten the dominant knee. Muscle strength was evaluated and recorded by giving values between 0 and 5 to the muscle resistance against gravity position and applied force. Previous research has revealed that manual muscle testing evaluation positively correlates with isokinetic dynamometry [26].

\section{Somatotype rating}

The somatotype is a dominance in the human body related to the concept of body shape, the evaluation methodology of which was first developed by Sheldon to define the variations in the human body. However, the method evolved by the Heath and Carter is now used to categorize the human physique based on the three-level scale, including endomorph, mesomorph, and ectomorph [3]. The somatotype components were determined based on the measurement of 16 parameters according to standard methods recommended by the International Society for the Advancement of Kinanthropometry [27]. Data from the anthropometric evaluations, including body height, values of the diameter, bicondylar width of femur and humerus, triceps, subscapular, supraspinal/ suprailiac and medial calf skinfold thickness (SF), arm and calf circumferences, body mass, were used to define characteristics of the human body (somatotype), by using Heath-Carter anthropometric somatotype method for anthropometric examination [28]. The skinfold thickness (SFs) was evaluated using the baseline skinfold caliper 12-1110 [29]. Knee and elbow widths were defined using the Holtain anthropometer set (Holstein Ltd., Crymych, Dyfed, Wales, UK). Weights were measured via Tanita body composition analyzer device (Tanita SC 330). Calf and arm circumferences were evaluated through the baseline circumference [30]. Finally, height was measured using the stadiometer (Denis -S200). Somatotype program v.1.2.6 established by Heath-Carter principles was utilized to describe somatotype values. According to the Heath-Carter classification system, the endomorphic component is characterized by a large subcutaneous fat deposit, while mesomorphic component describes a large bone mass and musculature. Finally, ectomorphic component signify a physique with slim or linear type [31]

\section{Statistical analysis}

All statistical analyses were carried out using the SPSS 25.0 package program. Descriptive data for the exercise capacity, somatotype components, BMI, FM, FFM, QF strength, and the ratio of each gender were given as percentage or mean and standard deviation, using descriptive statistics. Visual (histogram, probability plots), analytical method with ShapiroWilk test were performed to investigate whether or not that the variables were normally distributed. As well, the distortion or asymmetry of the data was investigated using kurtosis and skewness analyses. For these analyzes, a value range between 3 and +3 was accepted for skewness and a value range of 10 to +10 for kurtosis. As the measurements 'variables were either normally or non-normally distributed, both the Pearson and Spearman correlation coefficient (r) were used to reveal the strength of a linear association between exercise capacity and somatotype components, body composition, and quadriceps strength variables. Since the MET values were not normally distributed, the Mann-Whitney $U$ test was used to compare this parameter between quadriceps femoris strength of 4 and 5 . Finally, univariate, and multivariate linear regression analyses were performed to investigate the extent to which variables contributed to exercise capacity. A significance level of $p<0.05$ was used in the analysis of the data.

\section{Results}

Sixty patients with $\mathrm{CAD}$ (female $=12$ and male $=48$ ) were included in the study. Table 1 outlines participants' baseline characteristics, including age, percentage of gender, MET, anthropometric variables, somatotype components by gender, mean body fat, BMI, and QF strength.

\section{Table 1 Descriptive Data of Exercise Capacity, Somatotype Components, BMI, Body Fat, and Quadriceps Strength}

\begin{tabular}{|c|c|c|c|c|}
\hline \multicolumn{2}{|l|}{$n=60$} & \multicolumn{3}{|l|}{ Mean, SD } \\
\hline \multicolumn{2}{|c|}{$\begin{array}{l}\text { Age, years (Total) } \\
\text { Female }(\mathrm{n}=12) \\
\text { Male }(\mathrm{n}=48) \\
\text { Weight }(\mathrm{kg}) \\
\text { Height }(\mathrm{cm})\end{array}$} & \multicolumn{3}{|l|}{$\begin{array}{l}56.3 \pm 5.1 \\
57.7 \pm 3.01 \\
56.3 \pm 5.1 \\
86.3 \pm 14.4 \\
167.5 \pm 7.8\end{array}$} \\
\hline \multicolumn{2}{|c|}{$\begin{array}{l}\text { MET } \\
\text { (Min-Max) }\end{array}$} & \multicolumn{3}{|l|}{$10.8 \pm 1.8(7-13.5)$} \\
\hline \multicolumn{2}{|c|}{$\begin{array}{l}\text { Endomorph } \\
\text { (Min-Max) }\end{array}$} & \multicolumn{3}{|l|}{$\begin{array}{l}6.3 \pm 1.3 \\
(2.8-9)\end{array}$} \\
\hline \multicolumn{2}{|c|}{$\begin{array}{l}\text { Mesomorph } \\
\text { (Min-Max) }\end{array}$} & \multicolumn{3}{|c|}{$7.2 \pm 1.4(4.3-11.5)$} \\
\hline \multicolumn{2}{|c|}{$\begin{array}{l}\text { Ectomorph } \\
\text { (Min-Max) }\end{array}$} & \multicolumn{3}{|c|}{$0.5 \pm 0.7(-1.8-2.8)$} \\
\hline \multicolumn{2}{|c|}{$\begin{array}{l}\text { FFM (kg) } \\
\text { (Min-Max) }\end{array}$} & \multicolumn{3}{|c|}{$59.1 \pm 8.9(36.8-79)$} \\
\hline \multicolumn{2}{|c|}{$\begin{array}{l}\% \mathrm{FM} \\
\text { (Min-Max) }\end{array}$} & \multicolumn{3}{|c|}{$30.9 \pm 8.2(14.8-54)$} \\
\hline \multicolumn{2}{|c|}{$\begin{array}{l}\text { BMI }\left(\mathrm{kg} / \mathrm{m}^{2}\right) \\
(\text { Min-Max) }\end{array}$} & \multicolumn{3}{|c|}{$30,7 \pm 5,1(21,7-49,6)$} \\
\hline \multicolumn{2}{|c|}{$\begin{array}{l}\text { Quadriceps strength } \\
\text { (Min-Max) }\end{array}$} & \multicolumn{3}{|l|}{$4.4 \pm 0.5(4-5)$} \\
\hline \multicolumn{5}{|c|}{ Distribution of Somatotype Within Gender Group } \\
\hline & & $\begin{array}{l}\text { Endomorphic- } \\
\text { mesomorphic }\end{array}$ & $\begin{array}{l}\text { Mesomorphic- } \\
\text { endomorphic }\end{array}$ & Total \\
\hline \multirow[t]{2}{*}{ Gender } & Male & $27(56.3 \%)$ & $21(43.8 \%)$ & 48 \\
\hline & Female & $3(25.0 \%)$ & $9(75.0 \%)$ & 12 \\
\hline Total & & $30(50.0 \%)$ & $30(50.0 \%)$ & 60 \\
\hline
\end{tabular}

MET-Metabolic Equivalent; BMI-Body Mass Index; \% FM-Percentage of Fat Mass; FFM-Free-Fat Mass 


\begin{tabular}{|l|l|l|l|l|l|l|} 
& \%FM & FFM & BMI $\left(\mathrm{kg} / \mathrm{m}^{2}\right)$ & Endomorph & Mesomorph & Ectomorph \\
\hline Total $(\mathrm{n}=60)$ & $\mathrm{r}=-0.45$ & $\mathrm{r}=-0.12$ & $\mathrm{r}=-0.42$ & $\mathrm{r}=-0.39$ & $\mathrm{r}=-0.23$ & $\mathrm{r}=0.28$ \\
\hline Male $(\mathrm{n}=48)$ & $\mathrm{r}=-0.34$ & $\mathrm{r}=-0.28$ & $\mathrm{r}=-0.38$ & $\mathrm{r}=-0.34$ & $\mathrm{r}=-0.14$ & $\mathrm{r}=0.1$ \\
\hline Female $(\mathrm{n}=12)$ & $\mathrm{r}=-0.002$ & $\mathrm{r}=-0.29$ & $\mathrm{r}=-0.55$ & $\mathrm{r}=-0.19$ & $\mathrm{r}=-0.33$ & $\mathrm{r}=0.71$ \\
\hline
\end{tabular}

MET-Metabolic Equivalent; BMI-Body Mass Index; \% FM-Percentage of Fat Mass; FFM- Free-Fat Mass

\begin{tabular}{|c|c|c|c|c|c|c|}
\hline \multicolumn{2}{|c|}{ Table 3} & \multicolumn{5}{|c|}{$\begin{array}{l}\text { Comparison of Exercise Capacity (MET) by } \\
\text { Quadriceps Femoris (QF) Strength }\end{array}$} \\
\hline & QF: 4 & & QF: 5 & & $\mathrm{Z}$ & $\mathrm{P}$ \\
\hline \multirow{2}{*}{ MET } & Mean & SD & Mean & SD & \multirow{2}{*}{-5.458} & \multirow{2}{*}{0.000} \\
\hline & 9.76 & 1.55 & 12.21 & 1.13 & & \\
\hline
\end{tabular}

MET-Metabolic Equivalent; SD-Standard Deviation; QF-Quadriceps Femoris

According to BMI values, the study subjects were from normal weight to obese (range of the BMI: $21,7-49,6$ and mean BMI: 30,7 75,1$)$. In the male sample, 27 (56.3\%) presented with a body habit of endomorph-mesomorph, while $21(43.8 \%)$ were characterized as mesomorph-endomorph. Of the female participants, $3(25.0 \%)$ had an endomorph-mesomorphic body habit while $9(75.0 \%)$ had a mesomorph-endomorphic one. On the other hand, the distribution of physical habits in the total sample was the same $(50 \%)$. The participants of the study showed levels of both endomorph and mesomorph rather than ectomorph. In other words, endomorph and mesomorph were significantly dominant somatotype components compared with the ectomorph $(6.3 \pm 1.3 ; 7.2 \pm 1.4 ; 0.5 \pm 0.7)$

In the whole sample, exercise capacity was found to be negatively and weakly correlated with the endomorph and mesomorph components $(\mathrm{r} 1=-0.39$ and $\mathrm{r} 2=-0.23)$. In contrast, a positive and slight correlation was observed between the exercise capacity and ectomorph component $(\mathrm{r}=0.28)$. However, the correlations revealed between exercise capacity and somatotype components differentiated with gender-based analysis. For example, the relationship between exercise capacity and ectomorph component was trivial in male individuals $(\mathrm{r}=0.1)$, whereas significant in female individuals $(\mathrm{r}=0.71)$. Negatively moderate and weak correlations were found between exercise capacity and FM in the whole and male individuals $(\mathrm{r} 1=-0.45$ and $\mathrm{r} 2=-0.34$ ), respectively; on the other hand, there was no meaningful correlation between the same variables in the female individuals $(\mathrm{r}=-0.002)$. As for the correlation between exercise capacity and BMI, it was revealed a negative and moderate
Figure 1a - Box Plot of Exercise Capacity by Quadriceps Femoris Muscle Strength

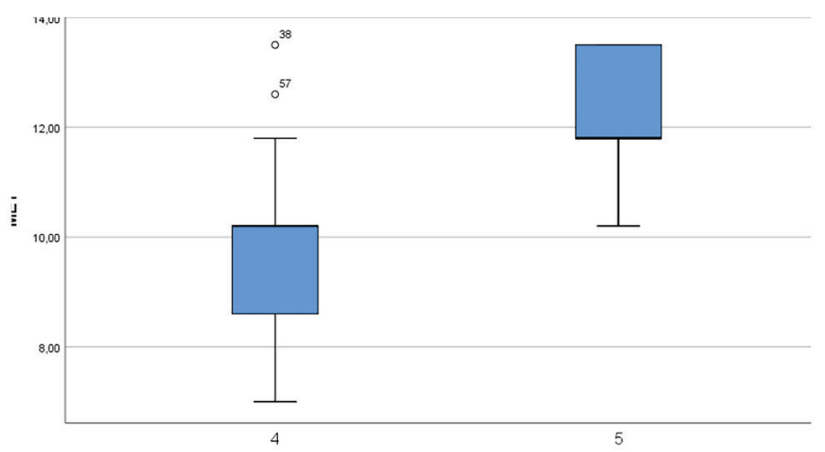

correlation between exercise capacity and BMI in the whole sample $(\mathrm{r}=-0.42)$. Based on the gender groups, exercise capacity was negatively and slightly correlated with the BMI in the male group $(r=-0.38)$ and moderately in the female group $(r=-$ 0.55). (Table 2). A statistically significant difference was found between the MET variables for different QF muscle strengths $(p<0.001)$ (Table 3). In other words, the mean MET values of individuals with muscle strength of 5 was significantly higher than those with muscle strength 4 (Figure 1).

Univariate regression analysis, conducted to investigate the influence of independent variables on exercise capacity separately, demonstrated that BMI, FM, and QF strength explained $\% 17, \% 20$, and $\% 44$ of variance in exercise capacity testing score, respectively. For the remainder of the dependent variables, it was not found out any remarkable variance explained. As for multivariate analyses, body composition elements, including FM, FFM and BMI, together explained a \% 24 of variance in exercise capacity testing score, while somatotype components together explained a \% 16 of variance in the exercise capacity. Finally, QF strength was found to be the best predictor of exercise capacity of patient with CAD $(\mathrm{R} 2=0.44$ or $\% 44)$ (Table 4$)$.

Univariate and Multivariate Regression Analyses on Exercise Capacity, Somatotype Components, Body Composition, and Quadriceps Strength

\begin{tabular}{|c|c|c|c|c|c|c|c|c|c|c|}
\hline \multicolumn{9}{|c|}{ Univariate Analysis } & \multicolumn{2}{|c|}{ Multivariate analysis } \\
\hline \multirow{8}{*}{ 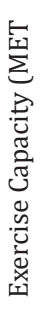 } & & $\mathrm{R}$ & $\mathrm{R}^{2}$ & $\mathrm{df}$ & $\mathrm{F}$ & $\mathrm{t}$ & $\mathrm{p}$-value & B & Adjusted $\mathrm{R}^{2}$ & $\mathrm{p}$-value \\
\hline & BMI & 0.42 & 0.17 & 1 & 12.405 & -3.522 & 0.001 & -0.15 & \multirow{3}{*}{0.24} & \multirow{3}{*}{0.001} \\
\hline & FM & 0.45 & 0.20 & 1 & 15.058 & -3.88 & 0.000 & -0.102 & & \\
\hline & FFM & 0.11 & 0.01 & 1 & 0.811 & 0.901 & 0.37 & 0.024 & & \\
\hline & Endomorph & 0.39 & 0.15 & 1 & 10.995 & -3.316 & 0.002 & -0.55 & \multirow{3}{*}{0.16} & \multirow{3}{*}{0.02} \\
\hline & Mesomorph & 0.23 & 0.05 & 1 & 3.287 & -1.813 & 0.07 & -0.288 & & \\
\hline & Ectomorph & 0.28 & 0.08 & 1 & 5.216 & 2.284 & 0.02 & 0.66 & & \\
\hline & $\mathrm{QF}$ & 0.66 & 0.44 & 1 & 46.607 & 6.827 & 0.000 & 2.452 & NA & NA \\
\hline
\end{tabular}

MET-Metabolic Equivalent; BMI-Body Mass Index; FM-Percentage of Fat Mass; FFM-Free-Fat Mass; QF-Quadriceps Femoris; NA-Not Applicable; R2Explained Variance (\%); df-Degree of Freedom

\section{Discussion}

In the whole sample, exercise capacity was negatively and slightly related to the endomorph and mesomorph components while positively and slightly related to the ectomorph component. Likewise, in the global approach, exercise capacity was found to be negatively and moderately correlated with fat mass and
BMI; on the contrary, no significant relationship was revealed between exercise capacity lean body mass. Body composition indices, including BMI, FM, and FFM, together explained \% 24 of variance in the exercise capacity testing score, whereas somatotype components explained \% 16 of variance in the exercise capacity testing score. 
As mentioned afore, as an atherosclerotic disease of the cardiovascular system, CAD has been reported one of the most causal factors of morbidity and mortality in both developed and developing countries [32]. Also, CAD has been demonstrated to be one of the essential factors of the disability [33]. Although advanced preventative and therapeutic approaches have significantly decreased the severity of the prognosis of cardiovascular diseases over the last decades [32]; however, $\mathrm{CAD}$ and related cardiovascular system diseases remain a vital risk of death in the global population. Physical bodily characteristics or body habitus, including fat-free mass, total body fat, subcutaneous fat pattern, somatotype (body shape), and relative weight and height (BMI measurements), have been reported as potential risk factors of cardiovascular diseases [3, 9]. Importantly, exercise or peak aerobic capacity was demonstrated to be a substantial prognostic factor in patients with or at risk of cardiovascular diseases [15, 34].

To date, many research focused on potential associations between somatotype components and cardiovascular diseases [3, 7, 35]. Furthermore, a previous study by Malina et al.[36] investigated possible relationships between somatotype components and cardiovascular risk factors in healthy adults, showing low to moderate relationships between these variables. As a result, considering that somatotype components are the important predictors of CAD [37], the varying degree of relationships between exercise capacity and somatotype components reported in this current study were not surprising. Marta et al.'s study [38] demonstrated that that the prevalence of coronary heart diseases in males was three times higher for endomorphic-mesomorphs than dominant ectomorphs. Similar to this result, we found that CAD was less relevant to ectomorph component than to endomorph-mesomorphic dyads. These additional results of our study confirmed that individuals characterized by ectomorphic dominance would be at a lower risk of coronary heart disease or CAD than their endomorphicmesomorphic peers. In other words, in this current study, the aerobic capacity of participants qualified with the ectomorphic component was found to be greater than those with endomorphicmesomorphic body habits. Another study [39] suggesting that the dominant somatotype affects exercise capacity and explosive strength in prepubescent children is closely related to our research results. The findings of a study demonstrating that the endomorph component negatively affects exercise capacity [40] are in line with these results of our study. In conclusion, considering that exercise capacity is a strong prognostic factor for cardiovascular diseases and mortality [15, 41, 42], it is vital to identify factors that negatively affect exercise capacity to improve or change them.

The modestly and inverse relationship between body fat percentage and exercise capacity in the current study can be attributed to the fact that a higher than normal body fat ratio may contribute less to energy production $[43,44]$. As well, the relationship between body fat and endomorph physique [39] confirm this meaningful relationship. As a result, the present results add to the evidence that increased fat mass is intimately related to with decreased exercise capacity [39, 45]. Gorant et al.[46] suggested that body fat did not influence VO2max or peak exercise capacity. However, our study results on the relationship between body fat and exercise capacity contradicted these study results. Surprisingly, this meaningful relationship between exercise capacity and body fat ratio in the general population differed by gender factor: While our study's findings show that body fat percentage negatively affects exercise capacity in men individuals, no such effect was found in women. This surprising result can be attributed to the small number of women in our study. Many researchers have reported that high BMI and increased body fat percentage in different populations adversely affect exercise capacity and physical fitness [47, 48]. Thus, a similar relationship exists in individuals with coronary artery disease, reinforcing that high BMI and increased body fat percentage predispose them to cardiovascular diseases. In this current study, it was found that knee extensor muscle strength was positively correlated with aerobic capacity. High lower extremity muscle strength paves the way for better exercise capacity; it results in less development of cardiovascular disease and consequently a lower risk of death. While the research on this subject mostly includes studies conducted on healthy individuals $[49,50]$, studies on individuals with coronary artery disease are extremely limited. Therefore, our study results reveal the relation between knee extensor muscle strength and right side in individuals with coronary artery disease to the limited literature. As a result, physical activity habits that increase physical fitness, as previously reported [24], are highly likely to reduce the risk of sudden cardiac death and acute myocardial infarction. It is critical in reducing coronary artery disease risk by keeping physical fitness parameters at an optimal level [51]. Also, a meta-analysis study conducted on this subject reported that Exercise-based cardiac rehabilitation reduced morbidity and mortality by $20-25 \%$ [52].

\section{Study limitations}

Despite its novel findings, our study may have several limitations. Firs, almost all the relationships between exercise capacity and dependent variables differentiated with genderbased statistical analysis. This may have very likely arisen from the small number of female patients. Later, all study participants were randomly recruited from the patients referred to the Cardiopulmonary Rehabilitation Unit of Trabzon Ahi Evren Thoracic and Cardiovascular Surgery Training and Research Hospital. Thus, further research is needed to investigate this subject in a broader population with CAD.

\section{Conclusion}

The current study was the first to explore the relationship between exercise capacity and body composition, somatotype components, and QF muscle strength in patients with CAD. Exercise capacity was found out to be negatively related to the endomorph and mesomorph components, suggesting that endomorphic-mesomorphs physique may negatively influence patients' aerobic capacity with CAD. In contrast, exercise capacity was revealed to be positively associated with the ectomorph physique, demonstrating that little fat mass may positively affect the exercise capacity. Likewise, in patients with CAD, exercise capacity was negatively and modestly associated with body fat mass and BMI, showing that lower body weight and adiposity in patients with CAD is essential. On the other hand, there was no meaningful relationship between exercise capacity and lean body mass. Finally, a significant and positive relationship between QF muscle strength and exercise capacity was revealed. In other words, quadriceps femoris strength might be a predictor of exercise capacity in patient CAD. Consequently, we suggested that somatotype components, adiposity, body composition, and QF muscle strength are meaningfully related to exercise capacity in the population with CAD. 
Disclosures: There is no conflict of interest for all authors.

Acknowledgements: The authors are gratitude to all study participants for their commitment to research timeline and requirements and Prof Dr. Gamze ÇAN for her valuable contribution. Since this current study featured a cross-sectional study, we have not required to obtain a clinical trial registration number. There is no financial support to report..

\section{Funding: None.}

Ethics approval: The study protocol was approved by the Ethical Committee of the XXX University for scientific research with the registration number of 24237859/187.
Consent to participate: Informed consent was obtained and signed by all patients.

Consent for publication: All the authors consent for publications.

Data sharing and data availability: The data that support the findings of this study are available from the corresponding author upon reasonable request.

\section{References}

1. Abubakar I, Tillmann T, Banerjee A. Global, regional, and national age-sex specific all-cause and cause-specific mortality for 240 causes of death, 1990-2013: a systematic analysis for the Global Burden of Disease Study. Lancet.2013; 385:117-171. doi: 10.1016/S01406736(14)61682-2. Epub 2014 Dec 18.

2. Members WG, Go AS, Mozaffarian D, Roger VL, Benjamin EJ, Berry JD, et al. Heart disease and stroke statistics—2013 update: a report from the American Heart Association. Circulation. 2013; 127:e6. doi: 10.1161/CIR.0b013e31828124ad.

3. Williams S, Jones E, Bell W, Davies B, Bourne M. Body habitus and coronary heart disease in men: A review with reference to methods of body habitus assessment. Eur Heart J.1997;18(3):376-93. doi: 10.1093/oxfordjournals.eurheartj.a015258.

4. Hajar R. Risk factors for coronary artery disease: historical perspectives. Heart Views. 2017;18(3):109-114. doi: 10.4103/HEARTVIEWS HEARTVIEWS 10617.

5. Girelli D, Martinelli N, Peyvandi F, Olivieri O. Genetic architecture of coronary artery disease in the genome-wide era: implications for the emerging "golden dozen" loci. Semin Thromb Hemost. 2009;35(7):671-82. doi: 10.1055/s-0029-1242721.

6. Rosengren A, Hawken S, Ônpuu S, Sliwa K, Zubaid M, Almahmeed WA, et al. Association of psychosocial risk factors with risk of acute myocardial infarction in 11119 cases and 13648 controls from 52 countries (the INTERHEART study): case-control study. Lancet. 2004; 11-177. doi: 10.1016/S0140-6736(04)17019-0.

7. Williams SR, Goodfellow J, Davies B, Bell W, McDowell I, Jones E. Somatotype and angiographically determined atherosclerotic coronary artery disease in men. Am J Hum Biol. 2000;12(1):128-138. doi: 10.1002/(SICI)1520-6300(200001/02)12:1<128::AIDAJHB14>3.0.CO;2-X

8. Ryan-Stewart H, Faulkner J, Jobson S. The influence of somatotype on anaerobic performance. PloS one. 2018 ;13(5):e0197761. doi: 10.1371/journal.pone.0197761.

9. Bastien M, Poirier P, Lemieux I, Després J-P. Overview of epidemiology and contribution of obesity to cardiovascular disease. Prog Cardiovasc Dis. 2014;56(4):369-81. doi: 10.1016/j.pcad.2013.10.016.

10. Pagidipati NJ, Zheng Y, Green JB, McGuire DK, Mentz RJ, Shah S, et al. Association of obesity with cardiovascular outcomes in patients with type 2 diabetes and cardiovascular disease: Insights from TECOS. Am Heart J. 2020;219:47-57. doi: 10.1016/j.ahj.2019.09.016.

11. Marta C, Marinho D, Costa A, Barbosa T, Marques M. Somatotype is more interactive with strength than fat mass and physical activity in peripubertal children. J Hum Kinet. 2011;29A:83-91. doi: 10.2478/v10078-011-0063-4.

12. Hillman C, Heinecke E, Hii J, Cecins N, Jenkins S, Eastwood P. Relationship between body composition, peripheral muscle strength and functional exercise capacity in patients with severe chronic obstructive pulmonary disease. Intern Med J. 2012;42(5):578-81. doi: 10.1111/j.1445-5994.2012.02771.x

13. Maeder M, Muenzer T, Rickli H, Brunner-La Rocca H, Myers J, Ammann P. How accurately are maximal metabolic equivalents estimated based on the treadmill workload in healthy people and asymptomatic subjects with cardiovascular risk factors? Int J Sports Med. 2008;29(8):658-63. doi: 10.1055/s-2007-989319.

14. Kozlov S, Caprnda M, Chernova O, Matveeva M, Alekseeva I, Gazdikova K, et al. Peak responses during exercise treadmill testing using individualized ramp protocol and modified Bruce protocol in elderly patients. Folia Med (Plovdiv). 2020; 31;62(1):76-81. doi: 10.3897/folmed.62.e49809.

15. Myers J, Prakash M, Froelicher V, Do D, Partington S, Atwood JE. Exercise capacity and mortality among men referred for exercise testing. N Engl J Med. 2002; 4;346(11):793-801. doi: 10.1056/NEJMoa011858.

16. Kamiya K, Mezzani A, Hotta K, Shimizu R, Kamekawa D, Noda C, et al. Quadriceps isometric strength as a predictor of exercise capacity in coronary artery disease patients. Eur J Prev Cardiol. 2014;21(10):1285-91. doi: 10.1177/2047487313492252.

17. Volaklis KA, Halle M, Meisinger C. Muscular strength as a strong predictor of mortality: a narrative review. Eur J Intern Med. 2015 ;26(5):303-10. doi: 10.1016/j.ejim.2015.04.013.

18. Rodgers GP, Ayanian JZ, Balady G, Beasley JW, Brown KA, Gervino EV, et al. American College of Cardiology/American Heart Association clinical competence statement on stress testing: a report of the American College of Cardiology/American Heart Association/American College of Physicians-American Society of internal medicine task force on clinical competence. Circulation. 2000;102(14):1726-38. doi: 10.1161/01.cir.102.14.1726.

19. Gibbons RJ, Balady GJ, Beasley JW, Bricker JT, Duvernoy WF, Froelicher VF, et al. ACC/AHA guidelines for exercise testing: a report of the American College of Cardiology/American Heart Association task force on practice guidelines (committee on exercise testing). $J$ Am Coll Cardiol. 1997;30(1):260-311. doi: 10.1016/s0735-1097(97)00150-2.

20. Banerjee A, Newman DR, Van den Bruel A, Heneghan C. Diagnostic accuracy of exercise stress testing for coronary artery disease: a systematic review and meta-analysis of prospective studies. Int J Clin Pract. 2012;66(5):477-92. doi: 10.1111/j.1742-1241.2012.02900.x.

21. Fletcher G, Ades P, Kligfield P, Arena R, Balady G, Bittner V,et al. Exercise standards for testing and training: a scientific statement from the American Heart Association. Circulation. 2013;128(8):873-934. doi: 10.1161/CIR.0b013e31829b5b44. Epub 2013 Jul 22.

22. Wang J, Thornton J, Kolesnik S, Pierson Jr. Anthropometry in body composition: an overview. Ann N Y Acad Sci.2000;904:317-26. doi: 10.1111/j.1749-6632.2000.tb06474.x. 
23. Engin B, Kutlubay Z, Yardımcı G, Vehid HE, Ambarcıŏlu P, Serdaroğlu S, et al. Evaluation of body composition parameters in patients with psoriasis. Int J Dermatol. 2014;53(12):1468-73. doi: 10.1111/ijd.12171.

24. Peterson CM, Thomas DM, Blackburn GL, Heymsfield SB. Universal equation for estimating ideal body weight and body weight at any BMI. Am J Clin Nutr. 2017;105(3):772. doi: 10.3945/ajen.116.151985.

25. Naqvi U, Sherman AL. Muscle Strength Grading. 3. In: StatPearls [Internet]. Treasure Island (FL): StatPearls Publishing; 2021 -. PMID: 28613779.

26. Bohannon RW. Manual muscle testing: does it meet the standards of an adequate screening test? Clin Rehabil. 2005;19(6):662-7. doi: 10.1191/0269215505cr873oa.

27. Ross W, Marfell-Jones MJ. Kinanthropometry. Physiological testing of the high-performance athlete. 1991; Accessed at:https://books. google.com.tr/books/about/Physiological_Testing_of_the_High_perfor.html?id=t98LAQAAMAAJ\&redir_esc=y

28. Yang LT, Wang N, Li ZX, Liu C, He X, Zhang JF, et al. Study on the adult physique with the Heath-Carter anthropometric somatotype in the Han of Xi'an, China. A Anat Sci Int. 2016;91(2):180-7. doi: 10.1007/s12565-015-0283-0. Epub 2015 May 5.

29. Norton K, Olds T. Anthropometrica: a textbook of body measurement for sports and health courses. UNSW press. Accessed at: https:// books.google.com.tr/books/about/Anthropometrica.html?id=0amPPwAACAAJ\&redir esc=y

30. Quiterio ALD, Carnero EA, Baptista FM, Sardinha LB. Skeletal mass in adolescent male athletes and nonathletes: relationships with high-impact sports. J Strength Cond Res. 2011;25(12):3439-47. doi: 10.1519/JSC.0b013e318216003b.

31. Bell W, Davies J, Evans W, Scanlon M, Mullen R. Somatic characteristics and cardiovascular risk factors in growth hormone deficiency: A randomized, double-blind, placebo-controlled study of the effect of treatment with recombinant human growth hormone. Am J Hum Biol : The Official Journal of the Human Biology Association. 2004;16(5):533-43. doi: 10.1002/ajhb.20055.

32. Malakar AK, Choudhury D, Halder B, Paul P, Uddin A, Chakraborty S. A review on coronary artery disease, its risk factors, and therapeutics. J Cell Physiol. 2019 Aug;234(10):16812-16823. doi: 10.1002/jcp.28350.

33. Gupta R, Guptha S, Sharma KK, Gupta A, Deedwania P. Regional variations in cardiovascular risk factors in India: India heart watch. World J Cardiol. 2012;4(4):112-20. doi: 10.4330/wjc.v4.i4.112.

34. Malik A, Kanduri JS, Asbeutah AAA, Khraishah H, Shen C, Welty FK. Exercise Capacity, Coronary Artery Fatty Plaque, Coronary Calcium Score, and Cardiovascular Events in Subjects With Stable Coronary Artery Disease. J Am Heart Assoc. 2020;9(7):e014919. doi: 10.1161/JAHA.119.014919.

35. Herrera H, Rebato E, Hernández R, Hernández-Valera Y, Alfonso-Sánchez M. Relationship between somatotype and blood pressure in a group of institutionalized Venezuelan elders. Gerontology. 2004;50(4):223-9. doi: 10.1159/000078351.

36. Malina RM, Katzmarzyk PT, Song TM, Theriault G, Bouchard C. Somatotype and cardiovascular risk factors in healthy adults. Am $J$ Hum Biol. 1997;9(1):11-19. doi: 10.1002/(SICI)1520-6300(1997)9:1<11::AID-AJHB3>3.0.CO;2-T.

37. Salimi HR, Heidari N, Salimi A. The relation between somatotype with aerobic capacity and balance in the boys 11-13 years. Turk $J$ Kin. 2016; 2(2): 23-26 Accessed at: https://dergipark.org.tr/tr/download/article-file/220318

38. Spain D, Nathan D, Gellis M. Weight, body type and the prevalence of coronary atherosclerotic heart disease in males. Am J Med Sci 1963 Jan;245:63-9. PMID: 13978493

39. Marta CC, Marinho DA, Barbosa TM, Carneiro AL, Izquierdo M, Marques MC. Effects of body fat and dominant somatotype on explosive strength and aerobic capacity trainability in prepubescent children. J Strength Cond Res. 2013;27(12):3233-44. doi: 10.1519/ JSC.0000000000000252.

40. Çinarli F, Kafkas M. The effect of somatotype characters on selected physical performance parameters. Physical education of students. 2019;23(6):279-87. https://doi.org/10.15561/20755279.2019.0602

41. Aslanger E, Assous B, Bihry N, Beauvais F, Logeart D, Cohen-Solal A. Association between baseline cardiovascular mechanics and exercise capacity in patients with coronary artery disease. Anatol J Cardiol 2016;16(8):608-613. doi: 10.5152/AnatolJCardiol.2015.6471. Epub 2015 Nov 18.

42. Chang J, Froelicher VF. Clinical and exercise test markers of prognosis in patients with stable coronary artery disease. Curr Probl Cardiol. 1994;19(9):533-87. doi: 10.1016/0146-2806(94)90010-8.

43. İri R, Yilmaz A, Aktuğ Z . The comparison of physical fitness levels and motoric features of elite footballers and handball players. Journal of Sports and Performance Researches. 2017; (1) 8:19-25. Accessed at: https://dergipark.org.tr/tr/download/article-file/1032809.

44. Tuan S, Su H, Chen Y, Li M, Tsai Y, Yang C, et al. Fat mass index and body mass index affect peak metabolic equivalent negatively during exercise test among children and adolescents in Taiwan. Int J Environ Res Public Health 2018 Feb 4;15(2):263. doi: 10.3390/ ijerph15020263.

45. Norman A-C, Drinkard B, McDuffie JR, Ghorbani S, Yanoff LB, Yanovski JA. Influence of excess adiposity on exercise fitness and performance in overweight children and adolescents. Pediatrics. 2005;115(6):e690-6. doi: 10.1542/peds.2004-1543.

46. Goran M, Fields D, Hunter G, Herd S, Weinsier R. Total body fat does not influence maximal aerobic capacity. Int J Obes Relat Metab Disord. 2000;24(7):841-8. doi: 10.1038/sj.ijo.0801241.

47. Willig AL, Hunter GR, Casazza K, Heimburger DC, Beasley TM, Fernandez JR. Body fat and racial genetic admixture are associated with aerobic fitness levels in a multiethnic pediatric population. Obesity (Silver Spring). 2011;19(11):2222-7. doi: 10.1038/oby.2011.

48. 48. Hsieh P-L, Chen M-L, Huang C-M, Chen W-C, Li C-H, Chang L-C. Physical activity, body mass index, and cardiorespiratory fitness among school children in Taiwan: a cross-sectional study. Int J Environ Res Public Health. 2014;11(7):7275-85. doi: 10.3390/ ijerph110707275.

49. 49. Vaara JP, Kyröläinen H, Niemi J, Ohrankämmen O, Häkkinen A, Kocay S,et al. Associations of maximal strength and muscular endurance test scores with cardiorespiratory fitness and body composition. J Strength Cond Res. 2012;26(8):2078-86. doi: 10.1519/ JSC. $0 \mathrm{~b} 013 \mathrm{e} 31823 \mathrm{~b} 06 \mathrm{ff}$.

50. Guclu MB, Ince DI, Arikan H, Savci S, Tulumen E, Tokgozoglu L. A comparison of pulmonary function, peripheral and respiratory muscle strength and functional capacity in the heart failure patients with different functional classes. Anadolu Kardiyol Derg. 2011;11(2):101-6. doi: 10.5152/akd.2011.027.

51. Bruning RS, Sturek M. Benefits of exercise training on coronary blood flow in coronary artery disease patients. Prog Cardiovasc Dis. 2015;57(5):443-53. doi: 10.1016/j.pcad.2014.10.006.

52. Almodhy M, Ingle L, Sandercock GR. Effects of exercise-based cardiac rehabilitation on cardiorespiratory fitness: A meta-analysis of UK studies. Int J Cardiol. 2016;221:644-51. doi: 10.1016/j.ijcard.2016.06.101. 